\title{
Smooth transition from sudden to adiabatic states in heavy-ion fusion reactions at deep-subbarrier incident energies
}

\author{
Takatoshi Ichikawa $^{1}$, Kouichi Hagino ${ }^{2}$, and Akira Iwamoto ${ }^{3}$ \\ 1 Yukawa Institute for Theoretical Physics, Kyoto University, Kyoto 606-8502, Japan \\ 2 Department of Physics, Tohoku University, Sendai 980-8578, Japan \\ 3 Advanced Science Research Center, Japan Atomic Energy Agency, Tokai-mura, Naka-gun, Ibaraki 319-1195, Japan
}

\begin{abstract}
We propose a novel extension of the standard coupled-channel (CC) model in order to account for the steep falloff of fusion cross sections at deep-subbarrier incident energies. We introduce a damping factor in the coupling potential in the CC model, simulating smooth transitions from sudden to adiabatic states in deepsubbarrier fusion reactions. The $\mathrm{CC}$ model extended with the damping factor can reproduce well not only the steep falloff of the fusion cross section but also the saturation of the logarithmic derivatives for the fusion cross sections at deep-subbarier energies for the ${ }^{16} \mathrm{O}+{ }^{208} \mathrm{~Pb},{ }^{64} \mathrm{Ni}+{ }^{64} \mathrm{Ni}$, and ${ }^{58} \mathrm{Ni}+{ }^{58} \mathrm{Ni}$ reactions at the deep-subbarrier energies.
\end{abstract}

\section{Introduction}

Channel couplings play an important role in heavy-ion fusion reactions at below the Coulomb barrier as a typical example of quantum tunneling of many-body systems. Because of a recent progress in experimental techniques, it has been possible to measure fusion cross sections down to deep-subbarrier incident energies [1-3]. These data show a substantial reduction of fusion cross sections at deepsubbarrier energies from the prediction of the CoupledChannel (CC) calculations that reproduce the experimental data at energies around the Coulomb barrier. This phenomenon, often referred to as the fusion hindrance, shows a threshold behavior, where the data deviate largely from the standard CC calculations at incident energies below a certain threshold energy, $E_{s}$.

An important aspect to understand the fusion hindrance is the density overlap of the colliding nuclei in the barrier penetration process. When the incident energy is below the barrier energy at the touching point of the colliding nuclei, $V_{\text {Touch }}$, the inner turning point of the potential is located inside the touching point, and the projectile is still in the classically forbidden region when the two colliding nuclei touch with each other (see Fig.1). In this situation, the colliding nuclei have to penetrate through a residual barrier with an overlapping configuration before fusion takes place. We will show below that $V_{\text {Touch }}$ calculated using several kinds of ion-ion potential indeed clearly correlates with experimentally determined threshold energy $E_{s}$ for many systems, indicating strongly that the nuclear interaction in the overlapping region plays an important role in the deep-subbarrier hindrance.

So far, three different theoretical approaches have been proposed in order to explain the fusion hindrance. Based on the sudden picture, Mişicu and Esbensen have intro- duced a repulsive core due to the Pauli exclusion principle to the nuclear interaction in the overlap region with the frozen-density approximation [4-6]. They obtained a much shallower potential pocket than the conventional one, resulting in the hindrance of the fusion probability due to the cutoff of high partial waves. In contrast, we have advocated the adiabatic approach by assuming neck formations between the colliding nuclei in the overlap region [12]. In our model, the fusion hindrance originates from the tunneling of much thicker potential barrier characterized by the adiabatic one-body potential. We extended the standard CC models in order to correctly treat the coupling potential in the case of the adiabatic approaches. This model achieved comparably good reproduction of the experimental data to the sudden model. The third approach, suggested recently by Dasgupta et al. [7], is the quantum decoherence of channel wave functions caused by the coupling to the thermal bath. A model calculation based on this picture shows a possibility of the gradual occurrence of hindrance in subbarrier fusion reactions $[8,9]$.

Among those three approaches, the origin of the deepsubbarrier hindrance considerably differs from each other. The recent precise data for the ${ }^{16} \mathrm{O}+{ }^{208} \mathrm{~Pb}$ fusion [7] may provide an adequate system to discriminate among the various models, because the behavior of its astrophysical Sfactor is difficult to reproduce with a simple model calculation. In the model of Esbensen and Mişicu, not only the collective inelastic channels but also the particle transfer channel with modified coupling strengths are necessary for a fit to the experimental data [6]. In the estimation of Dasgupta et al., it was impossible to obtain an overall fit to the experimental data from the above-barrier to deepsubbarrier regions with a single parameter set for the nuclear potential [7]. 


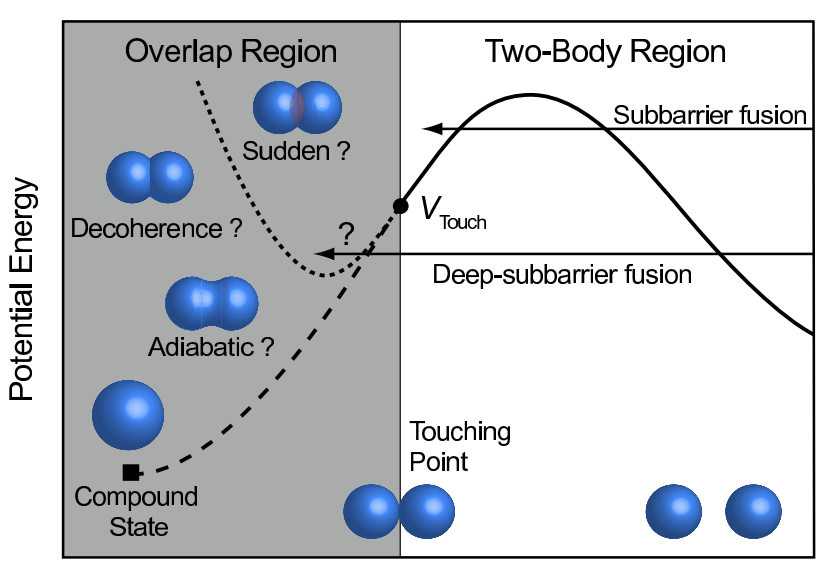

Center-of-Mass Distance $r$

Fig. 1. (Color online) Schematic picture of potential energy curve for heavy-ion fusion reactions. The gray area denotes the overlap region of two colliding nuclei.

On the other hand, our adiabatic model can reproduce well not only the hindrance of the fusion cross sections but also astrophysical S-factor and the adiabatic potential extracted from the experimental data in terms of the potential inversion method [10]. To achieve those, the standard CC model is modified to introduce the damping factor in the coupling potential, in order to simulate smooth transitions from the sudden to adiabatic states.

In this paper, we show the concept of the adiabatic approaches and propose an extension of the standard CC model in order to take into account the adiabatic potential full-quantum mechanically. We apply our model to the deep-subbarrier fusion for the ${ }^{16} \mathrm{O}+{ }^{208} \mathrm{~Pb},{ }^{58} \mathrm{Ni}+{ }^{58} \mathrm{Ni}$ and ${ }^{64} \mathrm{Ni}+{ }^{64} \mathrm{Ni}$ reactions and show the performance of our adiabatic model.

This paper is organize as follows: In Section 2, we discuss the correlation between the threshold energy and the energy at the touching point. In Section 3, we introduce the damping factor in order to describe the smooth transition from the sudden to adiabatic states. In Section 4, the calculated results are given. In Section 5, we summarize the paper.

\section{Correlation of threshold incident energy with energy at touching point}

We here discuss the correlation between the energy at the touching point and the threshold incident energy. In this respect, it is interesting to notice that the authors of Refs. [1] have argued that the steep fall-off phenomenon systematically takes place below a certain threshold incident energy, $E_{s}$. We evaluate the potential energy at the touching configuration for several systems, and compare those with $E_{s}$.

For the potential energy at the touching point, $r_{\text {touch }}=$ $R_{P}+R_{T}$, we here use the Yukawa-plus-exponential (YPE) model [11]. The calculated results of other potential models are given in Ref. [12]. Assuming the spherical shape for both the projectile and target nuclei, the YPE potential

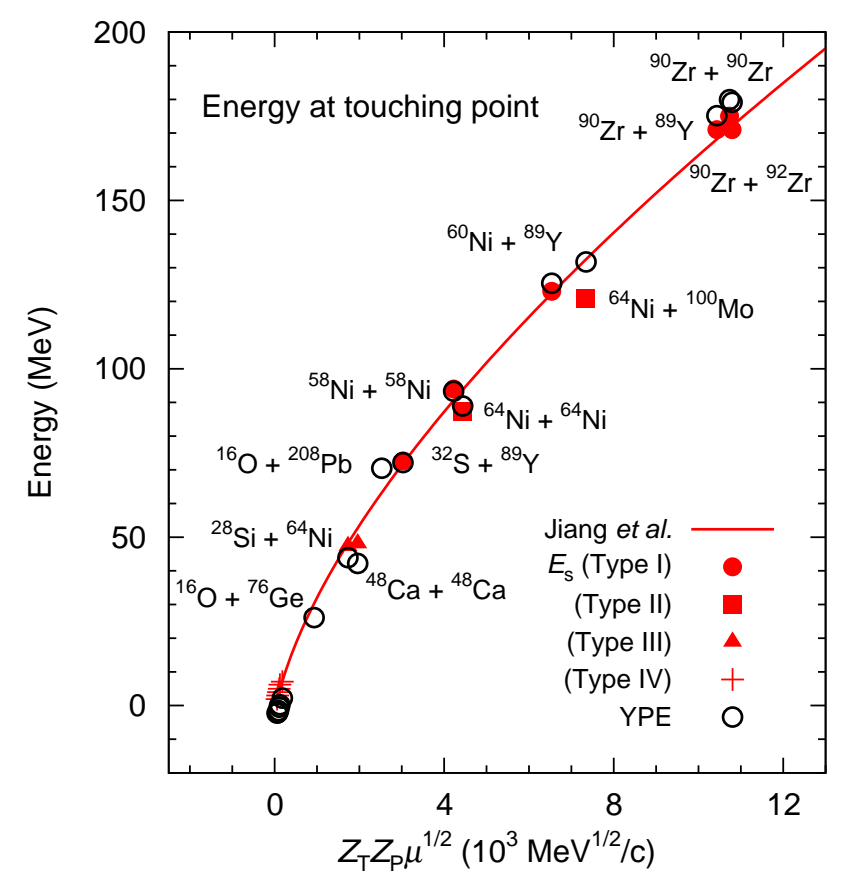

Fig. 2. (Color online) Potential energy at the touching point calculated with Yukawa-puls-exponential model. The open circle denotes the calculated results. The solid line denotes the systematics proposed by Jiang et al. [1]. The solid circles, squares, triangles, and the horizontal lines show the experimental energy taken from Ref. [1] at which the astrophysical S-factor has the maximum value.

energy at the touching point is given by

$$
V_{\mathrm{KNS}}^{(N)}=-D\left(4+\frac{r_{\text {touch }}}{a}-\frac{f\left(R_{T} / a\right)}{g\left(R_{T} / a\right)}-\frac{f\left(R_{P} / a\right)}{g\left(R_{P} / a\right)}\right),
$$

where the functions $f$ and $g$ are defined as $f(x)=x^{2} \sinh (x)$ and $g(x)=x \cosh (x)-\sinh (x)$, respectively. In this model, the nuclear radius is given by $R=r_{0} A^{1 / 3}$, and the depth constant $D$ by

$$
D=\frac{4 \sqrt{c_{s}^{(T)} c_{s}^{(P)}} a^{3}}{r_{0}^{2} r_{\text {touch }}} g\left(R_{T} / a\right) g\left(R_{P} / a\right) e^{-r_{\text {touch }} / a},
$$

where the effective surface energy constant $c_{s}$ is given by $c_{s}=a_{s}\left(1-\kappa_{s} I^{2}\right)$ with $I=(N-Z) / A$. We take the parameters to be $a=0.68 \mathrm{fm}, a_{s}=21.33 \mathrm{MeV}$ and $\kappa_{s}=2.378$ from FRLDM2002 [13], except for the radius parameter for which we slightly adjust to be $r_{0}=1.2 \mathrm{fm}$ in order to fit the experimental fusion cross sections for the ${ }^{64} \mathrm{Ni}+{ }^{64} \mathrm{Ni}$ reaction at energies above the Coulomb barrier.

To estimate the total potential energy at the touching point, $V_{\text {Touch }}$, one has to add the Coulomb potential to the nuclear potential energy given by Eq. 1. To this end, we use the Coulomb potential for two point charges, $V^{(C)}=$ $e^{2} Z_{T} Z_{P} / r_{\text {touch }}$. The resultant touching energy for the systems discussed in Ref. [1] is shown in Fig. 2 as a function of $Z_{T} Z_{P} \mu^{1 / 2}$, where $\mu$ is the reduced mass of the colliding nuclei.

The open circle denotes the calculated results of the YPE model. These touching energies are compared with 
the energy $E_{s}$, at which the experimental fusion cross section is maximum when it is plotted in terms of the astrophysical S-factor [1]. These "experimental" energies $E_{s}$ are shown in Fig. 2 by the filled circles, the filled squares, the filled triangles, and the horizontal lines, depending on the types of the system as defined in Ref. [1]. Notice that the energy $E_{s}$ for the type III was estimated by extrapolation, and that for the type IV is only an upper limit. The systematics for the energy $E_{s}$ proposed by Jiang et al. [1] is also shown by the solid line.

In the figure, we can clearly see that the calculated potential energies at the touching point follow the systematic curve proposed by Jiang et al., indicating that the dynamics after the touching point is responsible for the steep falloff phenomenon. We emphasize that our analysis is independent of the adiabatic or the sudden approaches because both provide a similar potential energy to each other as long as the touching point is concerned.

\section{Damping of coupling potential in coupled-channel model}

As was discussed in the previous section, the dynamics after the touching point plays an essential role on the steep falloff of the fusion cross section at the deep-subbarier incident energies. To describe those dynamics, we introduce an adiabatic process in the flamework of the standard CC model.

However, we can not directly apply the adiabatic potential to the standard CC model, because its direct application leads to the double counting of the $\mathrm{CC}$ effect. The channel coupling already includes many parts of the effects of the adiabatic process, such as the neck formations between the colliding nuclei. To avoid such double counting, we develop a full quantum mechanical model where the $\mathrm{CC}$ effect in the two-body system is smoothly jointed to the adiabatic potential tunneling for the one-body system.

We employ the incoming wave boundary condition in order to simulate a compound nucleus formation. In order to construct an adiabatic potential model with it, we postulate the followings: (1) Before the target and projectile nuclei touch with each other, the standard CC model in the two-body system works well. (2) After the target and projectile overlap appreciably with each other, the fusion process is governed by a single adiabatic one-body potential where the excitation on the adiabatic base is neglected. (3) The transition from the two-body treatment to the one-body one takes place at near the touching configuration, where all physical quantities are smoothly joined. To this end, we adopt Yukawa-plus-exponential (YPE) potential [11] as a basic ion-ion potential $V_{N}^{(0)}$, because the diagonal part of this potential satisfies the conditions (1)-(3) by choosing a suitable neck-formed shape for the one-body system, as has been shown in our previous work [12].

The nuclear coupling form factor which describes excitations to the vibrational states in the two-body channel is taken as the derivative of the internuclear potential
$V_{N}^{(0)}$ [14]. The coupling matrix elements are evaluated with the eigen-channel representation as in Eq. (24) in Ref. [15]. In order to satisfy the conditions (1)-(3), we employ the following form for the nuclear potential for the eigen-channel $\alpha$

$V_{N}\left(r, \lambda_{\alpha}\right)=V_{N}^{(0)}(r)+\left[-\frac{d V_{N}^{(0)}}{d r} \lambda_{\alpha}+\frac{1}{2} \frac{d^{2} V_{N}^{(0)}}{d r^{2}} \lambda_{\alpha}^{2}\right] \Phi\left(r, \lambda_{\alpha}\right)(3)$

where $\lambda_{\alpha}$ is the eigen value of the excitation operator. The most important modification from the standard CC treatment is the introduction of the damping factor $\Phi$. This damping factor represents the physical process for the gradual transition to the adiabatic approximation, by diminishing the strength of excitations to the target and/or projectile vibrational states after the two nuclei overlap with each other. We thus choose the damping factor given by

$$
\Phi\left(r, \lambda_{\alpha}\right)= \begin{cases}1 & r \geq R_{d}+\lambda_{\alpha} \\ e^{-\left(r-R_{d}-\lambda_{\alpha}\right)^{2} / 2 a_{d}^{2}} & (\text { Two-body region) } \\ & r<R_{d}+\lambda_{\alpha} \\ & \text { (Overlap region) },\end{cases}
$$

where $R_{d}$ is the spherical touching distance between the target and projectile defined by $R_{d}=r_{d}\left(A_{T}^{1 / 3}+A_{P}^{1 / 3}\right), r_{d}$ is the damping radius parameter, and $a_{d}$ is the damping diffuseness parameter. An important point of our modifications is that the touching point in the damping factor depends on $\lambda_{\alpha}$, that is, the strength of the excitations starts to decrease at the different distance in each eigen channel.

It is technically complicated to take into account the effect of the damping factor on the Coulomb coupling. We have introduced the channel independent damping factor for the Coulomb coupling, but the effect on the fusion cross sections appeared small. We therefore consider the damping factor only for the nuclear coupling in the calculations presented below.

\section{Results and discussions}

We apply our present model to the fusion reactions of the ${ }^{64} \mathrm{Ni}+{ }^{64} \mathrm{Ni},{ }^{58} \mathrm{Ni}+{ }^{58} \mathrm{Ni}$, and ${ }^{16} \mathrm{O}+{ }^{208} \mathrm{~Pb}$ systems. To this end, we incorporate the damping factor and the YPE potential in the computer code ccfull [15]. For the ${ }^{64} \mathrm{Ni}+{ }^{64} \mathrm{Ni}$ system, the coupling scheme included in the calculation, as well as the deformation parameters, are the same as in Ref. [16]. We treat ${ }^{64} \mathrm{Ni}$ as a spherical nucleus, although the effect of small, but finite, deformation was considered in Ref. [18]. For the ${ }^{58} \mathrm{Ni}+{ }^{58} \mathrm{Ni}$ system, those are the same as Ref. [14], but we include the coupling to the low-lying $2^{+}$and $3^{-}$phonon states and the triple-quadrupole and one octupole phonon excitations for both the target and projectile. We use a slightly large deformations of $\beta_{2}=0.23$ in the coupling potential. For the ${ }^{16} \mathrm{O}+{ }^{208} \mathrm{~Pb}$ system, those are the same as in Ref. [19], but we include the coupling to the low-lying $3^{-}$phonon states and the double-octupole phonon excitations for both the ${ }^{16} \mathrm{O}$ and ${ }^{208} \mathrm{~Pb}$ nuclei. For the damping factor, we use $r_{d}=1.298 \mathrm{fm}$ and $a_{d}=1.05 \mathrm{fm}$ 
for the ${ }^{64} \mathrm{Ni}+{ }^{64} \mathrm{Ni}$ system, $r_{d}=1.3 \mathrm{fm}$ and $a_{d}=1.3 \mathrm{fm}$ for the ${ }^{58} \mathrm{Ni}+{ }^{58} \mathrm{Ni}$ system, and $r_{d}=1.280 \mathrm{fm}$ and $a_{d}=1.28 \mathrm{fm}$ for the ${ }^{16} \mathrm{O}+{ }^{208} \mathrm{~Pb}$ system. Notice that the obtained damping radius parameters for the three systems which we study are almost the same.

The parameters of the YPE model are taken as $a_{0}=$ $0.68 \mathrm{fm}, a_{s}=21.33 \mathrm{MeV}$, and $\kappa_{s}=2.378$ from FRLDM2002 [13]. In order to fit the experimental fusion cross sections, the radius parameter $r_{0}$ is adjusted to be $1.205 \mathrm{fm}, 1.176 \mathrm{fm}$ and $1.202 \mathrm{fm}$ for the ${ }^{64} \mathrm{Ni}+{ }^{64} \mathrm{Ni},{ }^{58} \mathrm{Ni}+{ }^{58} \mathrm{Ni}$ and ${ }^{16} \mathrm{O}+{ }^{208} \mathrm{~Pb}$ systems, respectively. For the mass asymmetric system of ${ }^{16} \mathrm{O}+{ }^{208} \mathrm{~Pb}$, it is difficult to joint smoothly the potential energies between the two-body and the adiabatic one-body systems at the touching point, because the proton-to-neutron ratio for the one-body system differs from that for the target and projectile in the two-body system. To avoid this difficulty, we smoothly connect the potential energy around the touching point to the liquid-drop energy of the compound nucleus, using the third-order polynomial function (see the dashed line in Fig. 6). We do this by identifying the internucleus distance $r$ with the centers-of-masses distance of two half spheres. We have checked this prescription for the mass symmetric ${ }^{64} \mathrm{Ni}+{ }^{64} \mathrm{Ni}$ system, by comparing to the potential energy used in our previous work [12]. The deviation due to this prescription is negligibly small.

Figure 3 shows the fusion cross sections thus obtained. The fusion cross sections obtained with the damping factor are in good agreement with the experimental data for all the systems (see the solid line). For all the systems, we see that drastic improvement has been achieved by taking into account the damping of the CC form factors, as compared to the result without the damping factor (the dashed line).

We also compare the astrophysical $S$ factor representation of the experimental data with the calculated results, as shown in Fig. 4. In the calculation, the Sommerfeld parameter $\eta$ is shifted by $75.23,69.99$, and 49.0 for the ${ }^{64} \mathrm{Ni}+{ }^{64} \mathrm{Ni},{ }^{58} \mathrm{Ni}+{ }^{58} \mathrm{Ni}$, and ${ }^{16} \mathrm{O}+{ }^{208} \mathrm{~Pb}$ systems, respectively. The $S$ factor obtained with the damping factor are consistent with the experimental data for all the systems (see the solid lines), and reproduce well the peak structure. Notice that the $S$ factor predicted by our model differs considerably from that of the sudden model by Mişicu and Esbensen [5], denoted by the dot-dashed line at the lowest energies. For all the systems, as the incident energy decreases, their $S$ factor falls off steeply below the peak of the $S$ factor, while our $S$ factor has a much weaker energy dependence.

Figure 5 compares the logarithmic derivatives $d \ln \left(E_{\mathrm{c} . \mathrm{m}}\right.$. $\left.\sigma_{\text {fus }}\right) / d E_{\text {c.m. }}$ of the experimental fusion cross section with the calculated results. It is again remarkable that only the result with the damping factor achieves nice reproduction of the experimental data. For the ${ }^{64} \mathrm{Ni}+{ }^{64} \mathrm{Ni}$ and ${ }^{58} \mathrm{Ni}+{ }^{58} \mathrm{Ni}$ systems, the results with the damping factor becomes saturated below $E_{\text {c.m. }}=86 \mathrm{MeV}$ and $94 \mathrm{MeV}$, respectively. Those behaviors are similar to the experimental data for the ${ }^{16} \mathrm{O}$ $+{ }^{208} \mathrm{~Pb}$ system. The measurement at further lower incident energies for this system will thus provide a stringent test for the present adiabatic model.

Figure 6 shows the adiabatic potential of the ${ }^{16} \mathrm{O}+{ }^{208} \mathrm{~Pb}$ system, that is, the lowest eigenvalue obtained by diagonal-
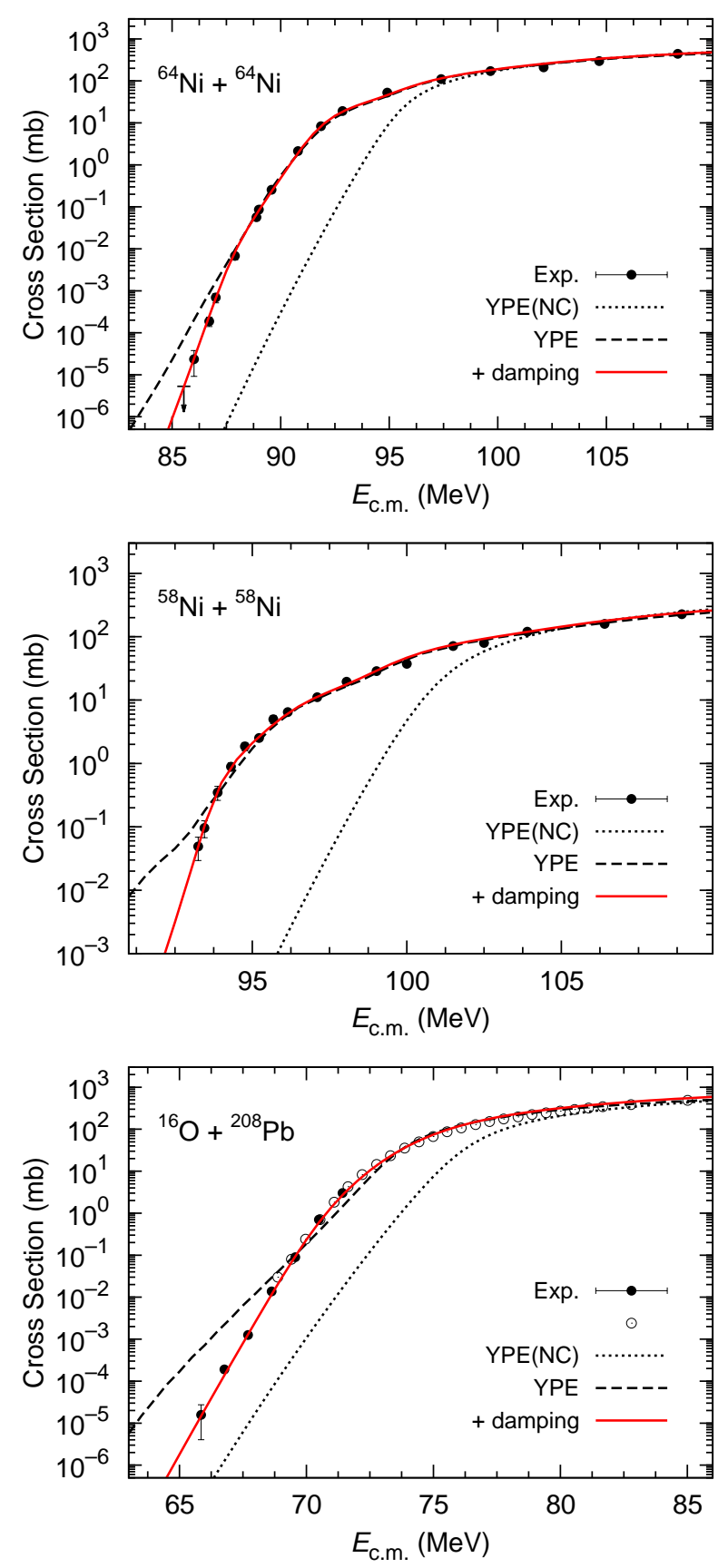

Fig. 3. (Color online) Fusion cross section calculated with the damping factor versus the incident energy for ${ }^{64} \mathrm{Ni}+{ }^{64} \mathrm{Ni}$ (top panel), ${ }^{58} \mathrm{Ni}+{ }^{58} \mathrm{Ni}$ (middle panel), and ${ }^{16} \mathrm{O}+{ }^{208} \mathrm{~Pb}$ (bottom panel). The solid and dashed lines denote the calculated result with and without the damping factor, respectively. The dotted line denotes the calculated result of the no coupling. The solid and open circles denote the experimental data taken from Refs. [1, 17,7].

izing the coupling matrix at each center-of-mass distance $r$. We see that the result obtained with the damping factor (the solid line) is much thicker than that of the conventional CC model (the dashed line). In this respect, it is interesting that the result with the damping factor is similar to that obtained with the potential inversion method [10], denoted by 

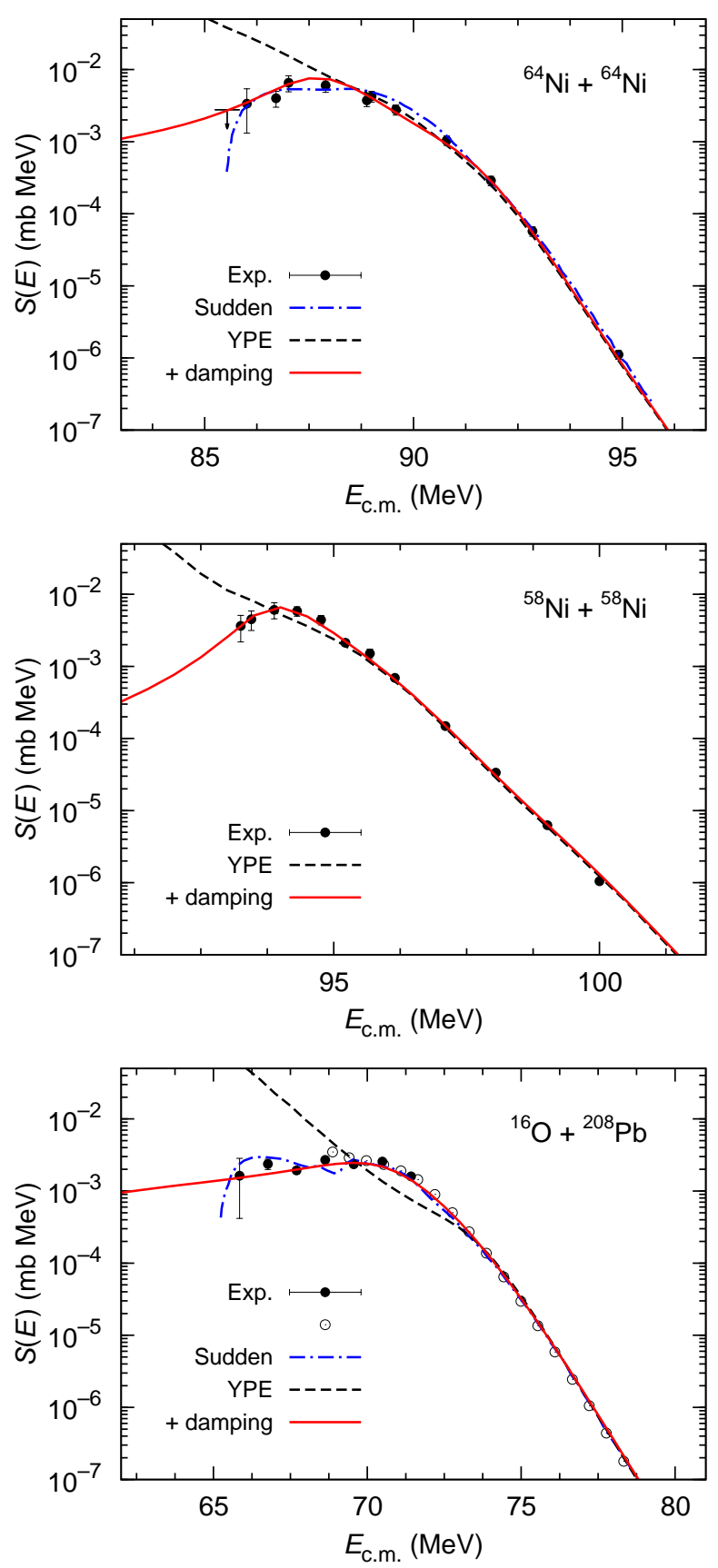

Fig. 4. (Color online) Astrophysical S-factor for the ${ }^{64} \mathrm{Ni}+{ }^{64} \mathrm{Ni}$ (top panel), ${ }^{58} \mathrm{Ni}+{ }^{58} \mathrm{Ni}$ (middle panel), and ${ }^{16} \mathrm{O}+{ }^{208} \mathrm{~Pb}$ (bottom panel) systems versus the incident energies. The meaning of each line is the same as in Fig. 3. The dot-dashed line is the result of the sudden model taken from Refs. [5] and [6].

the gray region, justifying our treatment for the damping of the $\mathrm{CC}$ form factor.

As long as fusion cross section is concerned, both the sudden approach of Mişicu and Esbensen [4] and our adiabatic approach provide similar results to each other. However, the origin of the fusion hindrance is different between the two approaches. In our model, the fusion hindrance takes place due to the smooth transition from the sudden to adiabatic potential (see also Ref. [12]). On the other hand,
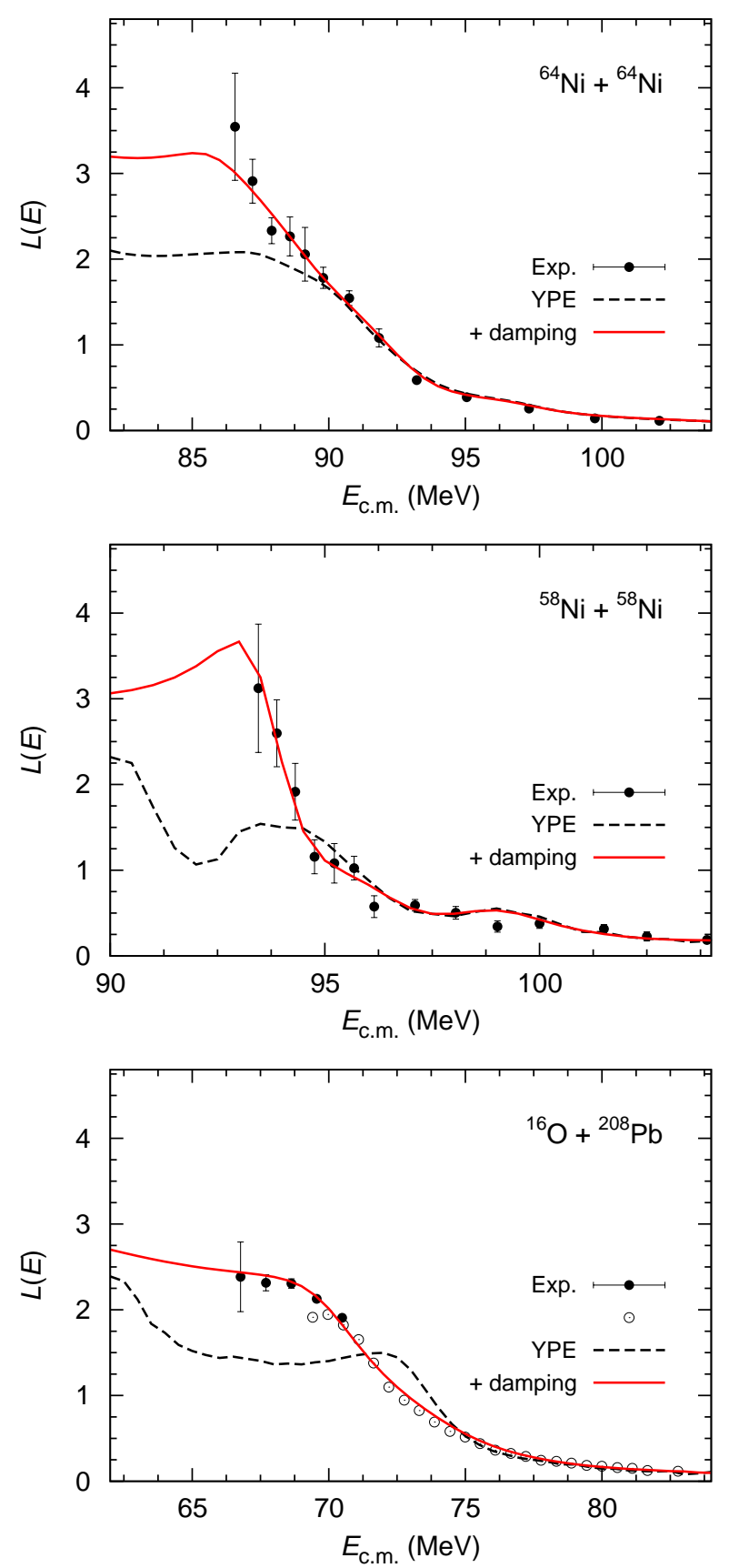

Fig. 5. (Color online) Logarithmic derivatives of fusion cross sections, $L(E)=d \ln \left(E_{\text {c.m. }} \sigma_{\text {fus }}\right) / d E_{\text {c.m. }}$, for the ${ }^{64} \mathrm{Ni}+{ }^{64} \mathrm{Ni}$ (top panel), ${ }^{58} \mathrm{Ni}+{ }^{58} \mathrm{Ni}$ (middle panel), and ${ }^{16} \mathrm{O}+{ }^{208} \mathrm{~Pb}$ (bottom panel) systems versus the incident energies. The meaning of each line is the same as in Fig. 3.

in the sudden model, which uses a shallow potential, the hindrance occurs because of the cut-off of the high angularmomentum components in the fusion cross section. The average angular momentum of the compound nuclei estimated by the sudden model therefore is much smaller than that of the present adiabatic model, as shown in Fig. 7. It is thus interesting to measure the average angular momentum of the compound nucleus at deep subbarrier energies, in order to discriminate between the two approaches. 


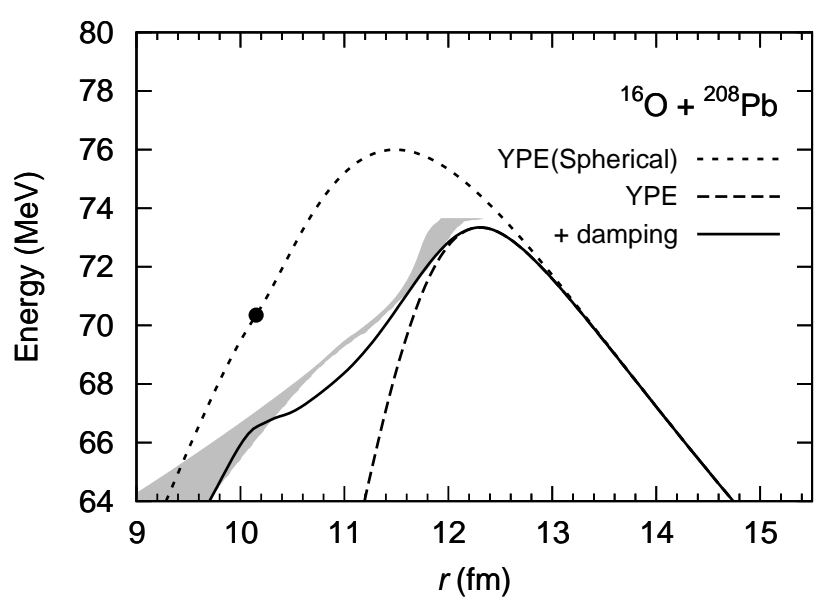

Fig. 6. The adiabatic potential for the ${ }^{16} \mathrm{O}+{ }^{208} \mathrm{~Pb}$ system versus the center-of-mass distance. The solid line is the adiabatic potential obtained with the damping factor. The dashed line is the result obtained with the conventional CC approach. The dotted line and the solid circle are the potential and the touching point for the uncoupled case, respectively. The gray region denotes the effective potential obtained with the potential inversion method, taken from Ref. [10].

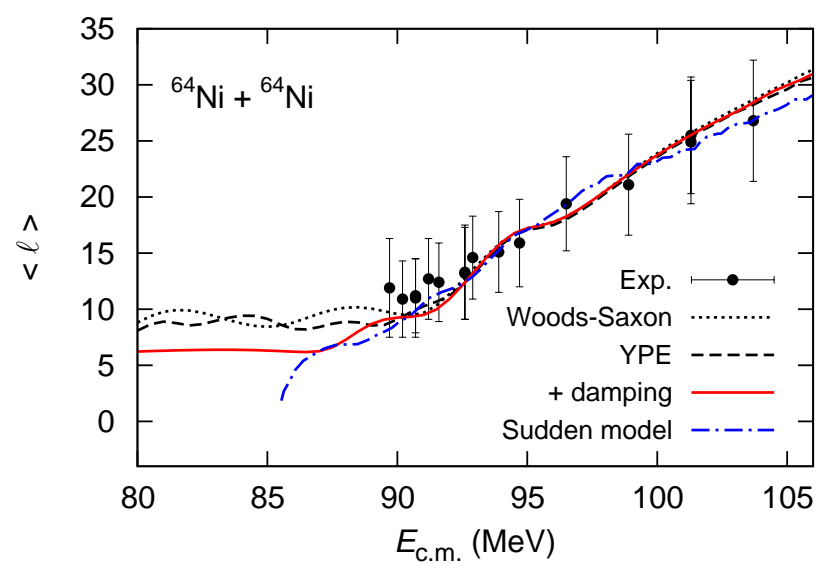

Fig. 7. (Color online) Calculated average angular momenta of the compound nuclei for the ${ }^{64} \mathrm{Ni}+{ }^{64} \mathrm{Ni}$ reaction. The solid line is the result of the present adiabatic model, while the dashed line is the result of the sudden model by Missicu and Esbensen taken from [8]. The dotted line is the result of the standard coupled-channel calculation with the Woods-Saxon potential.

\section{Summary}

In summary, we have proposed a novel coupled-channels approach for heavy-ion fusion reactions by introducing the damping of the $\mathrm{CC}$ form factor inside the touch point in order to simulate the transition from the sudden to adiabatic states. The important point in our present model is that the transition takes place at different places for each eigen channel. By applying this model to the ${ }^{16} \mathrm{O}+{ }^{208} \mathrm{~Pb}$, the ${ }^{64} \mathrm{Ni}+{ }^{64} \mathrm{Ni}$, and the ${ }^{58} \mathrm{Ni}+{ }^{58} \mathrm{Ni}$ systems, we conclude that the smooth transition from the two-body to the adiabatic one-body potential is responsible for the steep falloff of the fusion cross section.

\section{References}

1. C.L. Jiang et al., Phys. Rev. Lett. 89, (2002) 052701: Phys. Rev. C 69, (2004) 014604: Phys. Rev. C 71, (2005) 044613: Phys. Rev. C 73, (2006) 014613: Phys. Rev. C 75, (2007) 057604: Phys. Rev. C 78, (2008) 017601: Phys. Rev. C 79, (2009) 044601.

2. A.M. Stefanini et al., Phys. Rev. C 78, (2008) 044607: Phys. Lett. B 679, (2009) 95.

3. A. Shrivastava, Phys. Rev. Lett. 103, (2009) 232702.

4. Ş Mişicu and H. Esbensen, Phys. Rev. Lett. 96, (2006) 112701.

5. Ş. Mişicu and H. Esbensen, Phys. Rev. C 75, (2007) 034606 .

6. H. Esbensen and Ş. Mişicu, Phys. Rev. C 76, (2007) 054609.

7. M. Dasgupta et al., Phys. Rev. Lett. 99, (2007) 192701.

8. A. Diaz-Torres et al., Phys. Rev. C 78, (2008) 064604.

9. A. Diaz-Torres, Phys. Rev. C 81, (2010) 041603(R).

10. K. Hagino and Y. Watanabe, Phys. Rev. C 76, (2007) 021601(R).

11. H.J. Krappe, J.R. Nix, and A.J. Sierk, Phys. Rev. C 20, (1979) 992.

12. T. Ichikawa, K. Hagino, and A. Iwamoto, Phys. Rev. C 75, (2007) 057603.

13. P. Möller, A.J. Sierk, and A. Iwamoto, Phys. Rev. Lett. 92, (2004) 072501.

14. H. Esbensen and S. Landowne, Phys. Rev. C 35, (1987) 2090.

15. K. Hagino, N. Rowley, and A.T. Kruppa, Comput. Phys. Commun. 123, (1999) 143.

16. C.L. Jiang et al., Phys. Rev. Lett. 93, (2004) 012701.

17. M. Beckerman et al., Phys. Rev. C 25, (1982) 837

18. A.S. Umar and V.E. Oberacker, Phys. Rev. C 77, (2008) 064605: Eur. Phys. J. A 39, (2009) 243.

19. C.R. Morton et al., Phys. Rev. C 60, (1999) 044608.

20. T. Ichikawa, K. Hagino, and A. Iwamoto, AIP Conf. Proc. 1098, (2009) 32. 